\title{
THE WORK OF GEORGE ANDREWS: A MADISON PERSPECTIVE
}

BY

RiCHARD ASKEY

\section{Introduction.}

In his own contribution to this volume, George Andrews has touched on a number of themes in his research by looking at the early influences on him of Bailey, Fine, MacMahon, Rademacher and Ramanujan.

In this paper, I propose to present a survey of his work organized on a different theme. George has often alluded to the fact that his 1975-76 year in Madison was extremely important in his work. So it seems a reasonable project to survey his career from a Madison perspective. To make this story complete, I must begin in Sections 2-4 with Andrews' work in the late 1960's that led inexorably to our eventual lengthy collaboration. The year in Madison set in motion three seemingly separate strands of research that were fundamental in much of his subsequent work. These are described in Sections 5-8. Section 9 briefly describes his collaboration with Rodney Baxter. Section 10 describes the discovery of the crank. Section 11 contains a few concluding and summarizing comments.

\section{Partition Identities.}

From the time Rademacher taught him about the magic and mystery of the Rogers-Ramanujan identities, George was fascinated with such results. At first, he pursued them purely for their esthetic appeal. Rademacher [79; Lectures 7 and 8, pp. 68-84] presented the Rogers-Ramanujan identities as 
follows:

$$
\begin{aligned}
1+\frac{q}{1-q}+\frac{q^{4}}{(1-q)\left(1-q^{2}\right)} & +\frac{q^{9}}{(1-q)\left(1-q^{2}\right)\left(1-q^{3}\right)}+\cdots \\
& =\prod_{\substack{n>0 \\
n \equiv \pm(\bmod 5)}}^{\infty} \frac{1}{\left(1-q^{n}\right)} \\
1+\frac{q^{2}}{1-q}+\frac{q^{6}}{(1-q)\left(1-q^{2}\right)}+ & \frac{q^{12}}{(1-q)\left(1-q^{2}\right)\left(1-q^{3}\right)}+\cdots \\
& =\prod_{\substack{n>0 \\
n \equiv \pm 2(\bmod 5)}}^{\infty} \frac{1}{\left(1-q^{n}\right)}
\end{aligned}
$$

and he noted following MacMahon and Schur [79; pp. 69-72] that each may be interpreted partition - theoretically.

Identity (2.1) is equivalent to the assertion that the partitions of $n$ in which parts differ by at least 2 are equinumerous with the partitions of $n$ into parts congruent to \pm 1 modulo 5 .

Identity (2.2) is equivalent to the assertion that the partitions of $n$ into parts each $>1$ in which the parts differ by at least 2 are equinumerous with the partitions of $n$ into parts congruent to \pm 2 modulo 5 .

Rademacher [79; pp. 72-73] goes on to say

"The unexpected element in all these cases is the association of partitions of a definite type with divisibility properties. The leftside in the identities is trivial. The deeper part is the right side. It can be shown that there can be no corresponding identities for moludi higher than 5. All these appear as wide generalizations of the old Euler theorem in which the minimal difference between the summands is, of course, 1. Euler's theorem is therefore the nucleus of all such results."

Now while it may be argued that Rademacher was making a narrow statement here, it was taken by George to mean that there are no more results of this type.

So in his first year at Penn State much to his surprise, he discovered a generalization of the Rogers-Ramanujan identities by studying a paper of Selberg [83] which was quoted in a paper of Dyson [60] which in turn was quoted in Hardy and Wright [72]. Several months after his discovery, he learned that it had already been found by Basil Gordon [67]. 
Selberg based his extension of the Ramanujan-Rogers [80; pp. 214-215, 344-346] proofs of (2.1) and (2.2) on the following function

$$
C_{k i}(x ; q)=\sum_{n=0}^{\infty} \frac{(-1)^{n} x^{k n} q^{\frac{1}{2}(2 k+1) n(n+1)-i n}(x q ; q)_{n}\left(1-x^{i} q^{(2 n+1) i}\right)}{(q ; q)_{n}}
$$

where

$$
(a ; q)_{n}=(1-a)(1-a q) \cdots\left(1-a q^{n-1}\right) .
$$

George's proof [19; Ch. 7] of Gordon's generalization follows quite directly from the elegant functional equation found by Selberg [83]

$$
C_{k, i}(x ; q)-C_{k, i-1}(x ; q)=x^{i-1} q^{i-1}(1-x q) C_{k, k-i+1}(x q ; q) .
$$

However, what really got his attention was a review by W. Schwarz [82] of the Ph.D. thesis of Heinz Göllnitz [65]. The first paragraph of the review reads as follows:

"Continuing work of Schur [S.-B. Deutsch. Akad. Wiss. Munich 1926, 488-495] and Gleißberg [Math. Z. 28 (1928), 372-382], the author states about 15 theorems on partitions; for instance, the number of partitions of $n\left(n=a_{1}+a_{2}+\cdots, a_{1} \geqq a_{2} \geqq \cdots\right)$ into positive integers $a_{i} \equiv 2,5$ or $11(\bmod 12)$ is equal to the number of partitions of $n$ into different parts $b_{i} \equiv 2,4$ or $5(\bmod 6)$, and is equal to the number of partitions of $n$ into parts $c_{i} \neq 1,3$, where $c_{i}-c_{i+1} \geqq 6$ and $c_{i}-c_{i+1}>6$, if $c_{i} \equiv 0,1$ or 3 and $c_{i+1} \equiv 0,1$ or $3(\bmod 6)$."

It was easy enough for anyone to check this theorem for say $n \leqq 20$; however, George had no idea from his previous work how to prove this. So he wrote to Schwarz to see if he could obtain a copy of the Göllnitz thesis, and Schwarz sent him the review copy. This paper was challenging to say the least. The proof of the "mod 12" theorem alluded to above was elementary but absolutely overwhelming; it is a reasonably safe guess that no one (including George) has ever read it in detail. Indeed the study of the "mod 12" identity led to one stream of thought that has continued to this day and involved fruitful collaborations [2], [8], [9], [15], [16], [46]. However, the work in Göllnitz's thesis refers to work by Göllnitz undergraduate thesis [64] and that had the most profound impact on George's research. Namely 
Göllnitz proved the following identities: [64; p. 37]

$$
\begin{aligned}
& 1+q \frac{1+q}{1-q^{2}}+q^{4} \frac{(1+q)\left(1+q^{3}\right)}{\left(1-q^{2}\right)\left(1-q^{4}\right)} \\
& +q^{9} \frac{(1+q)\left(1+q^{3}\right)\left(1+q^{5}\right)}{\left(1-q^{2}\right)\left(1-q^{4}\right)\left(1-q^{6}\right)}+\cdots+q^{n^{2}} \prod_{k=1}^{n} \frac{1+q^{2 k-1}}{1-q^{2 k}}+\cdots \\
& =\prod_{n=0}^{\infty} \frac{1}{\left(1-q^{8 n+1}\right)\left(1-q^{8 n+4}\right)\left(1-q^{8 n+7}\right)} \\
& 1+q^{3} \frac{1+q}{1-q^{2}}+q^{8} \frac{(1+q)\left(1+q^{3}\right)}{\left(1-q^{2}\right)\left(1-q^{4}\right)} \\
& +q^{15} \frac{(1+q)\left(1+q^{3}\right)\left(1+q^{5}\right)}{\left(1-q^{2}\right)\left(1-q^{4}\right)\left(1-q^{6}\right)}+\cdots+q^{n(n+2)} \prod_{k=1}^{n} \frac{1+q^{2 k-1}}{1-q^{2 k}}+\cdots \\
& =\prod_{n=0}^{\infty} \frac{1}{\left(1-q^{8 n+3}\right)\left(1-q^{8 n+4}\right)\left(1-q^{8 n+5}\right)} .
\end{aligned}
$$

Göllnitz based his entire development on proving $q$-difference equations for the following function $[64 ;$ p. 37$]$

$$
\begin{aligned}
& \text { 2.8) } \quad G(s)=1=\sum_{n=1}^{\infty}(-1)^{n} s^{2 n} q^{n(4 n-1)}\left(1-s q^{4 n}\right) \\
& \times \frac{(1+q)\left(1+q^{3}\right)() \cdots\left(1+q^{2 n-1}\right)}{(1+s q)\left(1+s q^{3}\right) \cdots\left(1+s q^{2 n-1}\right)} \cdot \frac{\left(1-s q^{2}\right)\left(1-s q^{4}\right) \cdots\left(1-s q^{2 n-2}\right)}{\left(1-q^{2}\right)\left(1-q^{4}\right)\left(1-q^{6}\right) \cdots\left(1-q^{2 n}\right)} \\
& =1-s^{2} q^{3} \frac{\left(1-s q^{4}\right)(1+x)}{(1+s q)\left(1-q^{2}\right)}+s^{4} q^{14} \frac{\left(1-s q^{8}\right)(1+q)\left(1+q^{3}\right)\left(\left(1-s q^{2}\right)\right.}{(1+s q)\left(1+s q^{3}\right)\left(1-q^{2}\right)\left(1-q^{4}\right)} \\
& -s^{6} q^{33} \frac{\left(1-s q^{12}\right)(1+q)\left(1+q^{3}\right)\left(1+q^{5}\right)\left(1-s q^{2}\right)\left(1-s q^{4}\right)}{(1+s q)\left(1+s q^{3}\right)\left(1+s q^{5}\right)\left(1-q^{4}\right)\left(1-q^{6}\right)}+\cdots
\end{aligned}
$$

George saw that $G(s)$ and $C_{k, i}(x ; q)$ had to lie in a general family of such series. By extrapolating from these results, he was able [7] to provide a generalization of (2.6) and (2.7) (known as the Göllnitz-Gordon identities [64], [68]). From there he was led to a study of very-well-poised $q$-hypergeometric series [8] and their combinatorial implications [9], [15]. The culmination of this effort yielded the main theorem of [15].

Definition 1. If $\lambda$ is an even integer, we denote by $A_{\lambda, k, a}(n)$ the number of partitions of $n$ into parts such that no part $\not \equiv 0(\bmod \lambda+1)$ may be repeated and no part is $\equiv 0, \pm\left(a-\frac{1}{2} \lambda\right)(\lambda+1)(\bmod (2 k-\lambda+1)(\lambda+1))$. If $\lambda$ is an odd integer, we denote by $A_{\lambda, k, a}(n)$ the number of partitions of $n$ into parts such that no part $\not \equiv 0\left(\bmod \frac{1}{2}(\lambda+1)\right)$ may be repeated, no part is $\equiv \lambda+1(\bmod 2 \lambda+2)$, and no part is $\equiv 0, \pm(2 a-\lambda) \frac{1}{2}(\lambda+1)($ $\bmod (2 k-\lambda+1)(\lambda+1))$. 
Definition 2. Let $B_{\lambda, k, a}(n)$ denote the number of partitions of $n$ of the form $b_{1}+b_{2}+\cdots+b_{s}$, with $b_{i} \geqq b_{i+1}$, no parts $\not \equiv 0(\bmod \lambda+1)$ are repeated, $b_{i}-b_{i+k-1} \geqq \lambda+1$ with strict inequality if $(\lambda+1) \mid b_{i}$, and finally if $f_{j}$ denotes the number of appearances of $j$ in the partition, then $\sum_{i=j}^{\lambda-j+1} f_{i} \geqq a-j$ for $1 \leqq j \leqq \frac{1}{2}(\lambda+1)$, and $f_{1}+f_{2}+\cdots+f_{\lambda+1} \leqq a-1$.

Theorem. If $\lambda, k$, and $a$ are positive integers with $\lambda / 2<a \leqq k, k \geqq \lambda$, then

$$
A_{\lambda, k, a}(n)=B_{\lambda, k, a}(n)
$$

The Rogers-Ramanujan identities are the cases $\lambda=0, k=a=2$ and $\lambda=0, k=2, a=1$. Gordon's generalization is the case $\lambda=0$. George's generalization of Göllnitz-Gordon is $\lambda=1$, and his generalization of Schur's theorem in [14] is $\lambda=2$.

Ironically, H. Alder [1] proved that $B_{\lambda, 2,2}(n)$ is never equal to the number of partitions of $n$ taken from a fixed subset $S$ of the positive integers unless $\lambda \leqq 2$. The above theorem states clearly that while the particular generalization of Schur's theorem considered by Alder does not exist, there are indeed valid Gordon-like generalization of Alder's non-existence theorems for $B_{\lambda, k, a}(n)$. Indeed all that is necessary is that $k \geqq \lambda$.

This number-theoretic tour deforce made inevitable our meeting and collaboration. I too had been led into considering very-well-poised series of the ordinary (i.e. $q=1$ ) type. They arose naturally in the solution of the connection coefficient problem for Jacobi polynomials [55; p. 63], [56].

\section{More q-Series.}

By now, George was thoroughly engrossed in the study of $q$-series. His discovery that very well-poised series led to grand generalizations of the Rogers-Ramanujan identities caused him to look at a variety of $q$-series. In light of his work extending Watson's proof [85] of Ramanujan's fifth order mock theta function identities [4] [5] [6], George eventually considered $q$ Appell series. In a short paper [11], he showed that if

$$
\Phi^{(1)}\left[a ; b, b^{\prime} ; c ; x, y\right]=\sum_{n=0}^{\infty} \sum_{m=0}^{\infty} \frac{(a)_{m+n}(b)_{m}\left(b^{\prime}\right)_{n} x^{m} y^{n}}{(q)_{m}(q)_{n}(c)_{m+n}}
$$

and

$$
{ }_{r+1} \phi_{r}\left[\begin{array}{c}
a_{0}, a_{1}, \ldots, a_{r} ; q, z \\
b_{1}, \ldots, b_{r}
\end{array}\right]=\sum_{n=0}^{\infty} \frac{\left(a_{0}\right)_{n}\left(a_{1}\right)_{n} \cdots\left(a_{r}\right)_{n} z^{n}}{(q)_{n}\left(b_{1}\right)_{n} \cdots\left(b_{r}\right)_{n}}
$$


where

$$
(a)_{n}=(1-a)(1-a q) \cdots\left(1-a q^{n-1}\right),
$$

then

$$
\Phi^{(1)}\left[a ; b, b^{\prime} ; c ; x, y\right]=\frac{(a)_{\infty}(b x)_{\infty}\left(b^{\prime} y\right)_{\infty}}{(c)_{\infty}(x)_{\infty}(y)_{\infty}} \phi_{3}\left(\begin{array}{c}
c / a, x, y ; q, a \\
b x, b^{\prime} y
\end{array}\right)
$$

I found this result quite disturbing. At first glance, it is unreasonable. The Appell function $F^{(1)}[54$; Ch. IX] certainly does not reduce to an ordinary ${ }_{3} F_{2}$, and yet the above result asserts that a generalization of $F^{(1)}$ reduces to an ordinary $q$-hypergeometric function. Indeed, as George subsequently pointed out [18], all the known theorems for $\Phi^{(1)}$ are merely specializations of classical ${ }_{3} \phi_{2}$ transformations. It turns out that there is a satisfying and benign explanation of (3.4) as a $q$-integral which we both came to understand several years later during his visit to Madison in 1975-76. Namely, it is the $q$-analog of the integral representation of $F^{(1)}$.

In addition to this work, George was also considering $q$-series from the point of view of Rota's theory of functions of binomial type. Again he produced a study that disturbed me in quite a different way. In his paper on Eulerian differential operators [8], George suggests that there is probably no $q$-analog of the Rodrigues formula. His discussion was clearly inadequate to say the least; indeed, we found numerous $q$-Rodrigues formulas during the year he spent in Madison.

\section{The Evanston Meeting.}

Given the variety of ways that our interests converged (although starting from vastly different viewpoints), I wanted to get George and $q$-series involved in the world of special functions. The most immediate opportunity for this was the AMS Special Session on Special Functions that I was organizing for the regional meeting of the A.M.S. at Northwestern on April 27-28, 1973.

Inviting George to this meeting had several beneficial effects. First it did introduce him to many workers in special functions. Second, it induced him to prepare a SIAM Review survey article [16] out of the talk he gave; this was the first survey in a long time (if ever) that attempted to provide a variety of applications of $q$-series.

Finally, it provided one of those rare moments when one discovers that someone else shares one of your own pet peeves. For years I had been trying to point out that the rather confused world of binomial coefficient summations is best understood in the language of hypergeometric series identities. Time and again I would find first-rate mathematicians who had never heard 
of this insight and who would waste considerable time proving some apparently new binomial coefficient summation which almost always turned out to be a special case of one of a handful of classical hypergeometric identities.

To my great delight, George devoted a substantial portion of his talk to this exact topic. He even made the point more emphatically by illustrating that the hypergeometric understanding led naturally to the almost automatic construction of $q$-analogs. He finished his comments on this topic by asserting that $80 \%$ of the formulas in Table 3 of Henry Gould's Tables [70] yielded to this approach. This was the first portion of his talk with which I disagreed. I pointed out during the question period that the correct percentage was at least $90 \%$ if not $95 \%$. Independently, I had worked through the same chapter.

\section{The Madison Special Functions Meeting.}

After the Evanston meeting, George and I corresponded extensively, and I invited him to the CBMS conference in Blacksburg during June, 1974.

The following year I invited George to give one of the addresses at the MRC Advanced Seminar on Special Functions (March 31-April 2, 1975).

I had only heard George speak twice before; so I had reasonable confidence that he would give an interesting and comprehensible talk. He spoke in Session IV, at 3:15 pm on Tuesday, April 1, 1975. My confidence in his mathematical taste started to disintegrate immediately. His first slide read 


\section{Q-ANALOG OF EXTENDED MEIJER'S G-FUNCTION}

$$
\begin{aligned}
& G_{p, t, s, r}^{n, \nu_{1}, \nu_{2}, m_{1}, m_{2}}\left[\begin{array}{c}
x \\
y \\
\left(\gamma_{t}\right) ;\left(\gamma_{t}^{\prime}\right) \\
\left(\delta_{s}\right) \\
\left(\beta_{r}\right) ;\left(\beta_{r}^{\prime}\right)
\end{array} \mid q\right]= \\
& \sum_{h=1}^{m_{1}} \sum_{k=1}^{m_{2}} x^{\beta_{h}} y_{\beta_{k}^{\prime}} \frac{\prod_{j=\nu_{1}+1}^{t}\left(q / \gamma_{j} \beta_{h}\right)_{\infty} \prod_{j=\nu_{2}+1}^{t}\left(q / \gamma_{j}^{\prime} \beta_{k}^{\prime}\right)_{\infty}}{\prod_{j=1}^{\nu_{1}}\left(\gamma_{j} \beta_{h}\right)_{\infty} \prod_{j=1}^{\nu_{2}}\left(\gamma_{j}^{\prime} \beta_{k}^{\prime}\right)_{\infty}} \\
& \frac{\prod_{j=m_{1}+1}^{r}\left(q \beta_{h} / \beta_{j}\right)_{\infty} \prod_{j=m_{2}+1}^{r}\left(q \beta_{k}^{\prime} / \beta_{j}^{\prime}\right)_{\infty}}{m_{2}} \\
& \prod_{\substack{j=1 \\
j \neq k}}^{m_{2}}\left(\beta_{j}^{\prime} / \beta_{k}\right)_{\infty} \prod_{j=1}^{n}\left(q \beta_{h}^{\prime} \beta_{k}^{\prime} / \varepsilon_{j}\right)_{\infty} \\
& \frac{\prod_{j=n+1}^{p}\left(\varepsilon_{j} / \beta_{h} \beta_{k}^{\prime}\right)_{\infty} \prod_{j=1}^{s}\left(\delta_{j} \beta_{h} \beta_{k}^{\prime}\right)_{\infty}}{\prod_{\substack{j=1 \\
j \neq h}}^{m_{1}}\left(\beta_{j} / \beta_{h}\right)_{\infty}}
\end{aligned}
$$

To my horror a second slide was required just to complete this definition. Here is the content of the second slide:

$$
\Phi\left[\begin{array}{c|l|c}
p & \begin{array}{l}
\left(q \beta_{h} \beta_{k}^{\prime} / \varepsilon_{p}\right) \\
t
\end{array} & \begin{array}{l}
\left(\gamma_{t} \beta_{h}\right) ;\left(\gamma_{t}^{\prime} \beta_{k}^{\prime}\right) \\
\left(\delta_{s} \beta_{h} \beta_{k}^{\prime}\right) \\
\left(q \beta_{h} / \beta_{r}\right)_{h \neq r} ;\left(q \beta_{k}^{\prime} / \beta_{r}^{\prime}\right)_{k \neq r}+p-n+t-\nu_{1} x
\end{array} \\
r-1
\end{array} \mid\right.
$$

where

$$
\Phi\left[\begin{array}{c|l|c}
p & \\
t & \begin{array}{l}
\varepsilon_{1}, \varepsilon_{2}, \ldots, \varepsilon_{p} \\
\gamma_{1}, \gamma_{1}^{\prime}, \ldots, \gamma_{t}, \gamma_{t}^{\prime}
\end{array} & x \\
s & \delta_{1}, \delta_{2}, \ldots, \delta_{s} & \\
& \beta_{1}, \beta_{1}^{\prime}, \ldots, \beta_{r}, \beta_{r}^{\prime} & y
\end{array}\right]_{q}
$$




$$
=\sum_{m=0}^{\infty} \sum_{n=0}^{\infty} \frac{\left(\varepsilon_{1}\right)_{m+n} \cdots\left(\varepsilon_{p}\right)_{m+n}\left(\gamma_{1}\right)_{m}\left(\gamma_{1}^{\prime}\right)_{n} \cdots\left(\gamma_{t}\right)_{m}\left(\gamma_{t}^{\prime}\right)_{n} x^{m} y^{n}}{\left(\delta_{1}\right)_{m+n} \cdots\left(\delta_{s}\right)_{m+n}\left(\beta_{1}\right)_{m}\left(\beta_{1}^{\prime}\right)_{n} \cdots\left(\beta_{r}\right)_{m}\left(\beta_{r}^{\prime}\right)_{n}(q)_{m}(q)_{n}}
$$

where

$$
\begin{aligned}
(a)_{n} & =(a ; q)_{n}=(1-a)(1-a q) \cdots\left(1-a q^{n-1}\right), \\
(a)_{\infty} & =\lim _{n \rightarrow \infty}(a)_{n} .
\end{aligned}
$$

Had he lost his mind? This was the worst possible beginning of a talk on $q$-series that I could imagine. I started to hiss at him. Apparently he was waiting for this reaction, because the third slide followed saying:

\section{APRIL FOOL}

What a relief! His talk (without reference to these first 3 slides) appears in the proceedings of the conference [18]. It contained the seeds of several subsequent important research topics.

This is where the $q$-analog of the Dyson conjecture first appeared. Eventually Doron Zeilberger and David Bressoud [86] proved the conjecture. More importantly Ian Macdonald recognized the relationship of this conjecture with his own work [76] on identities, and he subsequently made much more general conjectures which have led to an explosion of research [77].

As bad as those first 3 slides were, George managed to put up a few more complicated formulas, such as Watson's $q$-analog of Whipple's theorem [57, p. 69]:

$$
\begin{aligned}
& { }_{8} \phi_{7}\left[\begin{array}{c}
a, g \sqrt{a},-q \sqrt{a}, b, c, d, e, q^{-N} ; q, X \\
\sqrt{a},-\sqrt{a}, \frac{a q}{b}, \frac{a q}{c}, \frac{a q}{d}, \frac{a q}{e}, a q^{N+1}
\end{array}\right] \\
= & \frac{(a)_{N}(a q / e f)_{N}}{(a q / e)_{N}(a q / f)_{N}} \phi_{3}\left[\begin{array}{l}
\frac{a q}{c d}, e, f, q^{-N} ; q, q \\
e f q^{-N} / a, \frac{a q}{c}, \frac{a q}{d}
\end{array}\right],
\end{aligned}
$$

where

$$
X=\frac{a^{2} q^{N+2}}{b c d e} .
$$

George presented the natural full generalization of this in [18], and this contains the basic mechanism of the Bailey chains that he first described fully in [26]. 
Namely, for $k \geqq 1, N$ a nonnegative integer,

$$
\begin{aligned}
{ }_{2 k+4} \phi_{2 k+3}\left[\begin{array}{c}
a, q \sqrt{a}, \\
\sqrt{a},-\sqrt{a}, a q / b_{1}, a q / c_{1}, a q / b_{2}, a q / c_{2}, \ldots, a q / b_{k}, a q / c_{k}, a q^{N+1}
\end{array}\right] \\
=\frac{(a q)_{n}\left(a q / b_{k} c_{k}\right)_{N}}{\left(a q / b_{k}\right)_{N}\left(a q / c_{k}\right)_{N}} \sum_{m_{1}, \ldots, m_{k-1}} \frac{\left(a q / b_{1} c_{1}\right)_{m_{1}}\left(a q / b_{2} c_{2}\right)_{m_{2}} \ldots\left(a q / b_{k-1} c_{k-1}\right)_{m_{k-1}}}{(q)_{m_{1}}(q)_{m_{2}} \ldots(q)_{m_{k-1}}} \\
\times \frac{\left(b_{2}\right)_{m_{1}}\left(c_{2}\right)_{m_{1}}\left(b_{3}\right)_{m_{1}+m_{2}}\left(c_{3}\right)_{m_{1}+m_{2}} \ldots}{\left(a q / b_{1}\right)_{m_{1}}\left(a q / c_{1}\right)_{m_{1}}\left(a q / b_{2}\right)_{m_{1}+m+2}\left(a q / c_{2}\right)_{m_{1}+m_{2}} \cdots} \\
\times \frac{\left(b_{k}\right)_{m_{1}+\cdots+m_{k-1}}}{\left(a q / b_{k-1}\right)_{m_{1}+\cdots+m_{k-1}}} \cdot \frac{\left(c_{k}\right)_{m_{1}+\cdots+m_{k-1}}}{\left(a q / c_{k-1}\right)_{m_{1}+\cdots+m_{k-1}}} \\
\times \frac{\left(q^{-N}\right)_{m_{1}+m_{2}+\cdots+m_{k-1}}}{\left(b_{k} c_{k} q^{-N} / a\right)_{m_{1}+m_{2} \cdots+m_{k-1}}} \\
\times \frac{(a q)^{m_{k-2}+2 m_{k_{3}}+\cdots+(k-2) m_{1}} q^{m_{1}+m_{2}+\cdots+m_{k-1}}}{\left(b_{2} c_{2}\right)^{m_{l}}\left(b_{3} c_{3}\right)^{m_{1}+m_{2}} \ldots\left(b_{k-1} c_{k-1}\right)^{m_{1}+m_{2}+\cdots+m_{k-2}}} .
\end{aligned}
$$

Now let's be honest. This proves George is an analyst. No one but a hard core analyst would have the nerve to extol a formula like this especially after his outrageous April Fool joke.

His comments [18; p. 205] about the work of Holman, Biedenham and Louck [75] caught the attention of Steve Milne (see for example [78]) who has subsequently in a work spaning two decades revealed what a rich theory was being hinted at in [75].

As is frequently the case, there was a sequel to his marvelous April Fool joke. A bit over a year ago, Doron Zeilberger sent out a three page paper titled "Mathematical Genitalysis: A Powerful New Combinatorial Theory that Obviates Mathematical Analysis." The abstract claimed that this new combinatorial theory would supersede and sometimes trivialize mathematical analysis, and illustrated this by an exact determination of Bloch's constant, and two other results. The last paragraph of the paper commented favorably on the NCTM Standards and Calculus Reform, and said that the new theory propounded in the note sent was consistent with these important movements.

George wrote me a note which said in part: "It is $100 \%$ certain that Doron sent this to you ... Whatever the merits of the sketchy body of the text, the last paragraph is vintage stuff."

My reply was to suggest that George look at the date this was posted on, April 1. He replied: "Why do I always fall for these things?" To which, my reply was: "Since you gave one of the best April Fool's jokes I have seen or heard, it is fitting that you get fooled at times." 


\section{The Year in Madison.}

By 1975, we were both aware of many areas of common interest. In particular each of us had looked seriously at the papers that Wolfgang Hahn had published in the late 1940's and early 1950's (see especially [71]). Each of us recognized that Hahn was pioneering a topic that was quite important. So I obtained money from the Mathematics Research Center and the Graduate School of the University of Wisconsin for George to spend the 1975-1976 academic year in Madison. Our plan which we followed fairly closely was to work through Hahn's paper [71].

This seminar led to our extended collaboration culminating many, many years later in the publication of Special Functions [42] (a project whose appearance would have never occurred without the resolute efforts of our co-author, Ranjan Roy).

In addition to our own collaboration, my two students, Jim Wilson and Dennis Stanton were introduced to $q$-series, and each has made substantial contributions to this topic.

The year (so George tells me) was one of the most fruitful of his career. He wrote and submitted The Theory of Partitions during that year. While he was writing Chapter 11 on plane partitions, I happened to mention to him a recent Russian book I had just obtained on the evaluation of determinants. This discussion reminded George of the tale he describes in Section 5 of [38], and simultaneously, unknown to me at the time, he was studying the BenderKnuth paper [59] which reduced MacMahon's then 75 year old conjecture on symmetric plane partitions to the following identity:

If

$$
g_{j}(q)=\prod_{i=1}^{s}\left\{\frac{\left(1-q^{j+2 i-1}\right)}{\left(1-q^{2 i-1}\right)} \prod_{h=i+1}^{s} \frac{\left(1-q^{2(j+i+h-1}\right)}{\left(1-q^{2(i+h-1}\right)}\right\}
$$

then

$$
g_{2 n}(q)=\operatorname{det}\left(C_{i-j}+C_{i+j-1}\right)_{n \times n},
$$

and

with

$$
g_{2 n+1}(q)=\left[\prod_{i=1}^{m}\left(1-q^{2 i-1}\right)\right] \operatorname{det}\left(C_{i-j}-C_{i+j}\right)_{n \times n}
$$

$$
C_{k}=q^{k^{2}}\left(\begin{array}{c}
2 m \\
m+k
\end{array}\right)_{2}
$$


$\left(\begin{array}{l}N \\ M\end{array}\right)_{r}$ is the Gaussian polynomial (or $q$-binomial coefficient) defined by $(6.4)$

$$
\left(\begin{array}{l}
N \\
M
\end{array}\right)_{r}= \begin{cases}\frac{\left(1-q^{N r}\right)\left(1-q^{(n-1) r}\right) \cdots\left(1-q^{(N-M+1) r}\right)}{\left(1-q^{M r}\right)\left(1-q^{(M-1) r}\right) \cdots\left(1-q^{r}\right)}, & 0<M \leqslant N \\
1, & M=0 ; \\
0, & M<0, M>N .\end{cases}
$$

This problem so consumed him that, apart from his appearances at our seminar, I saw almost nothing of him throughout October, 1975. Fortunately he was able to prove the MacMahon conjecture during that month [20], [22] and his interest in Hahn's paper resumed.

The seminar yielded a number of joint papers, for example [39], [40], and [41]. Probably [39] and [41] are most representative of our work. In [39] we developed the full solution of the connection coefficient problem for little $q$-Jacobi polynomials. Namely, if

then

$$
p_{n}(x ; \alpha, \beta \mid q)=\sum_{j=0}^{n} \frac{\left(q^{-n} ; q\right)_{j}\left(\alpha \beta q^{n+1} ; q\right)_{j} q^{j} x^{j}}{(q ; q)_{j}(\alpha q ; q)_{j}}
$$

where

$$
p_{n}(x ; \gamma, \delta \mid q)=\sum_{k=0}^{n} a_{k, n} p_{k}(x ; \alpha, \beta \mid q),
$$

$$
\begin{aligned}
a_{k, n}= & \frac{(-1)^{k} q^{k(k+1) / 2}\left(\gamma \delta q^{n+1} ; q\right)_{k}\left(q^{-n} ; q\right)_{k}(\alpha q ; q)_{k}}{(q ; q)_{k}(\gamma q ; q)_{k}\left(\alpha \beta q^{k+1} ; q\right)_{k}} \\
& \times \sum_{j=0}^{n-k} \frac{\left(q^{-n+k} ; q\right)_{j}\left(\gamma \delta q^{n+k+1} ; q\right)_{j}\left(\alpha q^{k+1} ; q\right)_{j} q^{j}}{(q ; q)_{j}\left(\gamma q^{k+1} ; q\right)_{j}\left(\alpha \beta q^{2 h+w} ; q\right)_{j}}
\end{aligned}
$$

From here it is a simple matter to deduce Watson's $q$-analog of Whipple's theorem (namely (5.1)). This was all based on the analogous results for the classical Jacobi polynomials [55; p. 63].

This inexorably led George to a mild generalization in [23] and eventually to the Bailey chains [26], a powerful method that has its genesis in a theorem of Bailey [58]. It was George's good fortune that Bailey was so diffident about his result that he only gave the recipe for it rather than displaying it and realizing its power.

In the next section, I shall describe the most bizarre event connected with George's year in Madison.

\section{Ramanujan's Lost Notebook.}

In the spring of 1976, Dominique Foata invited George to speak at the Table Ronde, Combinatoire et Représentation du Groupe Symétrique to 
be held in Strasbourg on April 26-30, 1976. George felt that his proof of MacMahon's conjecture was old news by now and that our joint work on $q$-analogs of the classical orthogonal polynomials was not ready for presentation.

He decided to prove the Bender-Knuth conjecture, a conjecture related to MacMahon's conjecture. Basil Gordon had announced a proof of the Bender-Knuth conjecture but did not publish anything on it until 1983 [69] and George assumed that his own methods would at least be novel. This was a dismaying prospect; I could envision him effectively disappearing for another month, if not more. Fortunately he took the approach of proving that Bender-Knuth and MacMahon were equivalent, a task that only took a few days [21].

As the time of the conference came closer, fate, in the guise of international airline fare irrationality, took a hand. In the spring of 1976, if you stayed in Europe at least 3 weeks, you could purchase a ticket for only a fraction of the amount required for any shorter stay. George asked for and received permission to stay in Europe for two weeks after the conference. He layed out an itinerary that included talks in Paris and Southampton, and a visit to Lucy Slater in Cambridge. Slater had told him about boxes deposited in the Trinity College Library which contained papers from the estate of the late G. N. Watson, the English analyst. This seemed at the time to be a rather minor aspect of his trip. Watson was a good analyst and had done good work (after all, he was the Watson of Whittaker and Watson); however, it would have been overly optimistic to expect to find much in these boxes. To his surprise, in one of the boxes was a $100+$ page manuscript in Ramanujan's inimitable handwriting [22].

In his own contribution to this volume, George has told the story of his discovery, so I won't repeat it here. I first found out about it the day he arrived back in Madison. "How did the trip go?" I asked. "Pretty well," he said. "I have in my briefcase, a Xerox of a 100+ page manuscript in Ramanujan's handwriting. I'm charging $25 \not \subset$ a peek!"

It would fill most of this volume if I were to recount in any detail the cornucopia of results that flowed from the Lost Notebook. George provided a survey in his introduction to the published version of the Lost Notebook [29] in 1988. Currently he and Bruce Berndt are collaborating on a fully edited version of the Lost Notebook.

A couple of summers later, George stopped in Madison on his way to the summer math meetings where he was to talk on the Lost Notebook. He gave a general talk and a specialized talk in Madison. At the second of these, there were six to eight people in the room and all of them knew a reasonable amount about q-series. I told George that this would probably be the largest audience he would have of people who knew a lot about basic hypergeometric series, so he could use the standard notation without fear. 
Little did either of us know how this field would develop, so that now an audience of 50 experts is not uncommon.

\section{The Mock Theta Function and Bailey Chain.}

I have already touched on the themes of Bailey chains and Ramanujan's Lost Notebook. In the early 80's, George started substantial use of computer algebra packages. This combined with the Bailey chains led to real breakthroughs in the study of mock theta functions.

The study of mock theta functions began with Ramanujan's last letter to Hardy in January, 1920, four months before he died. Here are a few excepts from that [80; pp. 354-355].

"If we consider a $\vartheta$-function in the transformed Eulerian form, e.g.,

$$
\begin{aligned}
& \text { (A) } 1+\frac{q}{(1-q)^{2}}+\frac{q^{4}}{(1-q)^{2}\left(1-q^{2}\right)^{2}}+\frac{q^{9}}{(1-q)^{2}\left(1-q^{2}\right)^{2}\left(1-q^{3}\right)^{2}}+\cdots \\
& \text { (B) } 1+\frac{q}{1-q}+\frac{q^{4}}{(1-q)\left(1-q^{2}\right)}+\frac{q^{9}}{(1-q)\left(1-q^{2}\right)^{2}\left(1-q^{3}\right)}+\cdots
\end{aligned}
$$

and determine the nature of the singularities at the points

$$
q=1, q^{2}=1, q^{3}=1, q^{4}=1, q^{5}=1, \ldots,
$$

we know how beautifully the asymptotic form of the function can be expressed in a very neat and closed exponential form. For instance, when $q=e^{-t}$ and $t \rightarrow 0$

$$
\begin{gathered}
(A)=\sqrt{\left(\frac{t}{2 \pi}\right)} \exp \left(\frac{\pi}{6 t}-\frac{t}{24}\right)+o(1), \\
(B)=\sqrt{\left(\frac{2}{5-\sqrt{5}}\right)} \exp \left(\frac{\pi}{15 t}-\frac{t}{60}\right)+o(1),
\end{gathered}
$$

and similar results at other singularities.

If we take a number of functions like (A) and (B), it is only in a limited number of cases the terms close as above; but in the majority of cases they never close as above. For instance, when $q=e^{-t}$ and $t \rightarrow 0$,

(C) $1+\frac{q}{\left(1-q^{2}\right)}+\frac{q^{3}}{\left(1-q^{2}\right)\left(1-q^{2}\right)^{2}}+\frac{q^{6}}{\left(1-q^{2}\right)\left(1-q^{2}\right)^{2}\left(1-q^{3}\right)^{2}}+\cdots$

$$
=\sqrt{\left(\frac{t}{2 \pi \sqrt{5}}\right)} \exp \left[\frac{\pi^{2}}{5 t}+a_{1} t+a_{2} t^{2}+\cdots+O\left(a_{k} t^{k}\right)\right],
$$


where $a_{1}=1 / 8 \sqrt{5}$, and so on. The function (C) is a simple example of a function behaving in an unclosed form at the singularities.

Now a very interesting question arises. Is the converse of the statements concerning the forms (A) and (B) true? That is to say: Suppose there is a function in the Eulerian form and suppose that all or an infinity of points are exponential singularities, and also suppose that at these points the asymptotic form of the function closes as neatly as in the cases of (A) and (B). The questions is: Is the function taken the sum of two functions one of which is an ordinary $\vartheta$-function and the other a (trivial) function which is $O(1)$ at all the ponts $e^{2 m \pi i / n}$ ? The answer is it is not necessarily so. When it is not so, I call the function a Mock $\vartheta$-function. I have not proved rigorously that it is no necessarily so. But I have constructed a number of examples in which it is inconceivable to construct a $\vartheta$-function to cut out the singularities of the original function. Also I have shown that if it is necessarily so then it leads to the following assertion-viz. it is possible to construct two power series in $x$, namely $\sum a_{n} x^{n}$ and $\sum b_{n} x^{n}$, both of which have essential singularities on the unit circle, are convergent when $|x|<1$, and tend to finite limits at every point $x=e^{2 r \pi i / s}$, and that at the same time the limit of $\sum a_{n} x^{n}$ at the point $x=e^{2 r \pi i / s}$ is equal to the limit of $\sum b_{n} x^{n}$ at the point $x=e^{-2 r \pi i / s}$.

This assertion seems to be untrue. Anyhow, we shall go to the examples and see how far our assertions are true."

Ramanujan concludes the letter with a list of mock theta functions together with identities satisfied by them. In G. N. Watson's LMS Presidential Address [84], he provides a method (relying on (5.1)) for a deep analysis of one collection of mock theta function (those that Ramanujan named "third order"). However Watson failed to produce an analysis of any comparable depth for the fifth order and seventh order functions.

In the Lost Notebook, George found a number of identities which would provide the missing analysis. For example, if $\phi_{0}(q)$ defined by

$$
\phi_{0}(q)=1+\sum_{n=1}^{\infty} q^{n^{2}}(1+q)\left(1+q^{3}\right) \cdots\left(1+q^{2 n-1}\right)
$$

is one of the fifth-order functions, then in the "lost" notebook we find a result equivalent to

$$
\begin{aligned}
\phi_{0}(-q) & =\prod_{n=0}^{\infty} \frac{\left(1-q^{5 n+5}\right)\left(1+q^{5 n+2}\right)\left(1+q^{5 n+3}\right)}{\left(1-q^{10 n+2}\right)\left(1-q^{10 n+8}\right)} \\
+1 & -\sum_{n=0}^{\infty} \frac{q^{5 n^{2}}}{(1-q)\left(1-q^{6}\right) \cdots\left(1-q^{5 n+1}\right)\left(1-q^{4}\right)\left(1-q^{9}\right) \cdots\left(1-q^{5 n-1}\right)}
\end{aligned}
$$


It follows immediately by an application of (5.1), that

$$
\begin{aligned}
& \phi_{0}(-q)=\prod_{n=0}^{\infty} \frac{\left(1-q^{5 n+5}\right)\left(1+q^{5 n+2}\right)\left(1+q^{5 n+3}\right)}{\left(1-q^{10 n+2}\right)\left(1-q^{10 n+8}\right)} \\
& +1-\prod_{n=0}^{\infty}\left(1-q^{5 n+5}\right)^{-1}\left\{\frac{1}{1-q}+\left(1-q^{-1}\right) \sum_{n=1}^{\infty} \frac{(-1)^{n} q^{n(15 n+5) / 2}\left(1+q^{5 n}\right)}{\left(1-q^{5 n+1}\right)\left(1-q^{5 n-1}\right)}\right\} .
\end{aligned}
$$

This and similar identities for the other fifth order mock theta functions were central to their study as George noted [22].

The key to unlocking such formulas lay in a subtle application of the Bailey chain. George has given an account of the basic properties of the chain in Some Debts I Owe; so I will restrict myself to one portion of the study. Namely the objects he calls Bailey pairs. Sequences of functions $\left\{\alpha_{n}\right\}$ and $\left\{\beta_{n}\right\}$ satisfying

$$
\beta_{n}=\sum_{r=0}^{n} \frac{\alpha_{r}}{(q ; q)_{n-r}(q ; q)_{n+r}}
$$

The pair key to the understanding of $\phi_{0}(q)$ is

$$
\beta_{n}=\frac{(-1)^{n} q^{-n(n-1) / 2}}{(q ; q)_{n}}
$$

and

$$
\alpha_{n}=q^{n^{2}+n} \sum_{j=-n}^{n}(-1)^{j} q^{-j(3 j+1) / 2}-q^{n^{2}-n} \sum_{j=1-n}^{n-1}(-1)^{j} q^{-j(3 j+1) / 2}
$$

The form of $\alpha_{n}$ is sufficiently surprising not to mention complicated that without the help of SCRATCHPAD (a.k.a. AXIOM) to compute many $\alpha_{n}$ 's, these discoveries never would have occurred.

From this point on, Dean Hickerson played a vital role eventually proving all the Mock Theta conjectures [73] and proving comparable theorems for the seventh order functions [74].

\section{Physics.}

George's collaborations with physicists began with Rodney Baxter and Peter Forrester in [46]; the resulting model, generalizing Baxter's Hard Hexagon Model, is succinctly called the ABF Model.

The mathematics background that George brought to bear on this is best laid out in his only single author physics publication [25]. Here he observes 
that a number of the functions produced by the method of Corner Transfer Matrices are limits of nice polynomials. The prototype example is Schur's theorem [81].

If $D_{0}=D_{1}=1$, and $D_{n}=D_{n-1}+q^{n-1} D_{n-2}$ for $n \geqq 2$, then

$$
D_{n}=\sum_{\lambda=-\infty}^{\infty}(-1)^{\lambda} q^{\lambda(5 \lambda+1) / 2}\left[\begin{array}{c}
n \\
\left\lfloor\frac{n-5 \lambda}{2}\right\rfloor
\end{array}\right]
$$

where $\lfloor x\rfloor$ is the greatest integer in $x$ and $\left[\begin{array}{l}A \\ B\end{array}\right]$ is the familiary Gaussian polynomial defined in (6.4).

George had already studied generalizations of Schur's theorem in [12] and [13]. It turned out that the ABF model could be treated by an analysis of polynomials similar in nature to Schur's polynomial version of the RogersRamanujan identities.

In a subsequent collaboration with Baxter [45], they discover $q$-analogs of the trinomial numbers. The latter are the entries in the following table where each entry is the sum of the 3 entries directly above it

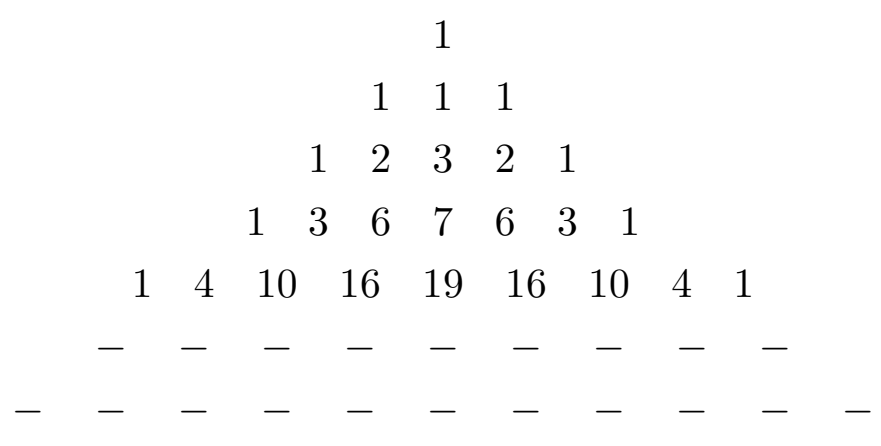

An example of the polynomials in question is $[43 ;$ p. 299]

$$
\left(\begin{array}{c}
n ; B ; q \\
A
\end{array}\right)_{2}=\sum_{j \geqq 0} \frac{q^{j(j+B)}(q ; q)_{n}}{(q ; q)_{j}(q ; q)_{j+A}(q ; q)_{n-2 j-A}}
$$

Besides solving the model with Baxter in [43], [44] and [45], George found a variety of applications including a full explanation of a mathematical mystery that had been called by Euler: "A remarkable example of misleading induction." [31]. More recently he has worked with physicists A. Berkovich [47] and A Schilling and O. Warnaar [54] on further extensions of the Bailey chain. 


\section{The Crank.}

There are many sides to George Andrews. The one which will probably have the most long lasting impact is the problem solver. His ability in this regard can be illustrated in many ways. Here is one instance of what happened when he felt challenged in an area that most of us think of as his.

Ramanujan discovered a number of very surprising congruences for the number of partitions of some infinite families of numbers. There were three infinite families, with the first of each family being the following:

$$
\begin{aligned}
& p(5 n+4) \equiv 0 \quad(\bmod 5) \\
& p(7 n+5) \equiv 0 \quad(\bmod 7) \\
& p(11 n+6) \equiv 0 \quad(\bmod 11)
\end{aligned}
$$

For the first two of these congruences, Freeman Dyson [61] discovered a combinatorial reason for the existence of these facts, and his conjecture was proven by Atkin and Swinnerton-Dyer [3]. The statistic found by Dyson did not work in the third case, so he expressed a hope that another statistic could be found. These names were appropriate, "rank" for the one he found, and "crank" for the one still undiscovered one. Over 40 years later, Frank Garvan, one of George's Ph.D. students, found a pseudo crank, and talked about this at the Ramanujan meeting in Urbana in 1987. Garvan felt that there was a real version of this unknown statistic, and told a number of people about an identity which he felt was the key.

George was struck by Frank's observation that the pseudo crank generating function

$$
\prod_{n=1}^{\infty} \frac{\left(1-q^{n}\right)}{\left(1-z q^{n}\right)\left(1-z^{-1} q^{n}\right)}
$$

has only one negative coefficient (that of $z^{0} q^{1}$ ). Previously they had assumed that the appearance of one negative coefficient suggested that negative coefficients would abound. Since the specialization $z=1$ in (10.1) produced

$$
\prod_{n=1}^{\infty} \frac{1}{1-q^{n}}=\sum_{n \geqq 0} p(n) q^{n}
$$

Frank's observation suggests strongly that the pseudo crank generating function might indeed generate the crank at least for $n>1$.

On Saturday, June, 1987, the day after the Ramanujan meeting, George undertook a melancholy journey to Onarga, Illinois to visit the graves of his parents (who were born in the mid-west and chose to be buried there 
even though they spent all their adult lives in Oregon). He returned to Urbana in the early afternoon in a very somber mood. He hoped to discuss mathematics with a few people who had remained after the conference, but no one was around in the dormitory where he was staying. To pull himself out of the doldrums he sat down to study (10.4) with the hope of tracking down the crank.

By the early evening, he had found the crank [49; p. 168].

Now he faced a nonmathematical problem. He was low on cash, had no phone card and only had access to pay phones. How could he let Frank know? He proceeded to call his wife collect. "I want you to call Richard Askey who will know Frank Garvan's phone number. I want you to say that I would like to write a joint paper with Frank in which we show that the crank of a partition is given by: [he then read the definition of the crank symbol by symbol]."

This provided the first crank. It should be noted that further cranks (i.e. partition statistics that provide combinatorial interpretations of (10.3) were found by Garvan, Kim and Stanton, [63] and that Dyson [62] provided many further insights about the Garvan-Andrews crank.

\section{Conclusion.}

It would be incorrect to conclude that the bulk of George's research concluded in the 1980's. Indeed a full account of his work in the 1990's would perhaps require another paper comparable to this one. I shall only mention a few of his themes refer you to the literature. It's not clear which of his current projects will have staying power similar to those I have already described. Also the breadth of projects widens partially due to the fact that he is undertaking many more collaborations now than in the past; indeed 27 of his 44 lifetime collaborators have written papers with him in the 1990's.

His interest in partition identities sparked a continuing collaboration with K. Alladi that began with [2].

The Liouville mystery [37] (referred to in Some Debts I Owe) is essentially work on generalized Lambert series and relates to his work with Crippa and Simon [30].

His Pfaff Trilogy as he calls it ([34], [35] and [36]) is both part of his continuing interest in computer algebra methods and applications to plane partitions.

The papers with the Knopfmachers (beginning with [50]) on generalizations of the Engel expansion suggest a try new line of research. The idea here is to produce an algorithm to expand an infinite product or modular form into an Eulerian or $q$-series. If this method can be expanded it may well take its place as perhaps the converse of Euler's ancient algorithm [19; p. 98] for obtain infinite product representations of generating functions. 
Also in this volume we find one of his papers (joint with P. Paule) [52] on the computer algebra implementation of the Partition Analysis of P. A. MacMahon. This project clearly points beyond MacMahon's horizon in that the authors have observed and implemented the fact that Partition Analysis is a purely algorithmic process. The success of Geroge's work on the Omega Package (joint with Paule and Riese [51], [52], [53]) suggests a number of further refinements and applications.

There is much more to the story and I have probably left out a number of George's favorite items. This is to be expected. The topics that interested me most are the ones I can most easily discuss.

I want to express my thanks to George. First, he provided some of the details in this paper. I remember the start of his April 1 lecture in Madison, but not the specific forumlas which were given above. He provided them and some other facts. More importantly, he taught me about basic hypergeometric series. My mathematical life would have been significantly different without his teaching. There are quite a few people who could say the same, their mathematical life would have been poorer without the aid which George Andrews provided them. 


\section{REFERENCES}

1. H. L. Alder, The nonexistence of certain identities in the theory of partitions and compositions, Bull. Amer. Math. Soc. 54 (1948), 712-722.

2. K. Alladi, G. E. Andrews and B. Gordon, Generalizations and refinements of a partition theorem of Göllnitz, J. reine und angew. Math. 460 (1995), 165-188.

3. A. O. L. Atkin and H. P. F. Swinnerton-Dyer, Some properties of partitions, Proc. London Math. Soc. (3) 4 (1953), 84-106.

4. G. E. Andrews, On basic hypergeometric series, mock theta functions, and partitions, I, Quart. J. Math. 17 (1966), 64-80.

5. _ On basic hypergeometric series, mock theta functions, and partitions, II, Quart. J. Math. 17 (1966), 132-143.

6. _ q-identities of Auluck, Carlitz, and Rogers, Duke Math. J. 33 (1966), 575582.

7. A generalization of the Gollnitz-Gordon partition theorems, Proc. Amer. Math. Soc. 8 (1967), 945-952.

8. __ On q-difference equations for certain well-poised basic hypergeometric series, Quart. J. Math. 19 (1968), 433-447.

9. , A generalization of the classical partition theorems, Trans. Amer. Math. Soc. 145 (1968), 205-221.

10. - On the foundations of combinatorial theory, V.: Eulerian differential operators, Studies in Applied Math 50 (1971), 345-375.

11. Summations and transformation for basic Appell series, J. London Math. Soc. 4(2) (1972), 618-622.

12. Sieves for theorems of Euler, Rogers and Ramanujan, Lecture Notes in Mathematics \#251, Springer-Verlag, NY, (1972) pp. 1-20.

13. __ Sieves in the theory of partitions, Amer. J. Math. 94 (1972), 1214-1230.

14. __ Partition identities, Advances in Math. 9 (1972), 10-51.

15. _ On the general Rogers-Ramanujan theorem, Memoir. Amer. Math. Soc. 152 (1974), 86.

16. Applications of basic hypergeometric functions, SIAM Rev. 16 (4) (1974), 441-484.

17. An analytic generalization of the Rogers-Ramanujan identities for odd moduli, Proc. Nat. Acad. Sci. 71 (10) (1974), 4082-4085.

18. __ Problems and prospects for basic hypergeometric functions, Theory and Application of Special Functions, R. Askey, ed., Academic Press, NY, (1975) pp. 191214.

19. The Theory of Partitions, The Encyclopedia of Mathematics and Its Applications Series, Addison-Wesley Pub. Co., NY, (1976) pp. 300. Reissued, Cambridge University Press, New York, 1998.

20. _ MacMahon's conjecture on symmetric plane partitions, Proc. Nat. Acad. Sci. 74 (2) (1977), 426-429.

21. _ Plane partitions, II: The equivalence of the Bender-Knuth and MacMahon conjectures, Pacific J. Math. 72 (2) (1977), 283-291. 
22. __ An introduction to Ramanujan's "lost" notebook, Amer. Math. Monthly 86 (2) (1979), 89-108.

23. C_ Connection coefficient problems and partitions, Proc. Symp. in Pure Math. 34 (1979), 1-24.

24. _ Plane partitions, III: The weak Macdonald conjecture, Invent. Math. 53 (1979), 193-225.

25. - The hard-hexagon model and the Rogers-Ramanujan type identities, Proc. Natl. Acad. Sci. 78 (1981), 5290-5292.

26. __ Multiple series Rogers-Ramanujan type identities, Pac. J. Math. 114 (2) (1984), 267-283.

27. 293 (1) (1986), 113-134.

28. _ Plane partitions, IV: A conjecture of Mills-Robbins-Rumsey, Aequationes Mathematicae 33 (1987), 230-250.

29. Introduction to Srinivasa Ramanujan: The Lost Notebook and Other Unpublished Papers, Narosa, New Delhi (1987).

30. _ Mock theta functions, Proc. Symposia in Pure Math. 49 (1989), 283-298.

31. Euler's "Exemplum Memorabile Inductionis Fallacis" and q-trinomial coefficients, J. Amer. Math. Soc. 3 (1990), 653-669.

32. __ Bailey chains and generalized Lambert series, I: Four identities of Ramanujan, Illinois J. Math. 36 (1992), 251-274.

33. , Plane partitions, V: The T.S.S.C.P.P. conjecture, J. Combinatorial Th. (A) 66 (1) (1994), 28-39.

34. _ Pfaff's Method (II): Diverse applications, J. Computational \& Appl. Math. 68 (1996), 15-23.

35. __ Pfaff's Method (III): Comparison with the WZ method, Electronic J. Combin. (Foata Festschrift) 3 (2): R21 (1996), 18.

36. , Pfaff's Method (I): The Mills-Robbins-Rumsey determinant, Discrete Math. 193 (1998), 43-60.

37. _ Three-quadrant Ferrers graphs, (submitted to volumes for the B. N. Prasad Centenary Commemoration, Allahabad Math. Society).

38. __ Some debts I owe, (submitted to Proceedings of 42nd Séminaire Lotharingien de Combinatoire, held in my honor at Maratea, Italy, September, 1998).

39. (with R. Askey), Enumeration of partitions: The role of Eulerian series and q-orthogonal polynomials, Higher Combinatorics, M. Aigner, ed., Reidell Publ. Co., Dordrecht, Holland, (1977) pp. 3-26.

40. (with R. Askey), A simple proof of Ramanujan's ${ }_{1} \psi_{1}$ summation, Aequa. Math. 18 (1978), 333-337.

41. (with R. Askey), Another q-extension of the $\beta$-function, Proc. Amer. Math. Soc. 81 (1) (1981), 97-100.

42. _ (with R. Askey), Classical orthogonal polynomials, Proc. Conf. on Orthog. Polys. at Bar-le duc, Lecture Notes in Math., Springer, Berlin, 1171 (1985) pp. 3662.

43. (with R. J. Baxter), Lattice gas generalization of the hard hexagon model, I: Star triangle relation and local densities, J. Stat. Phys. 44 (1/2) (1986), 259-271.

44. (with R. J. Baxter), Lattice gas generalization of the hard hexagon model. II. The local densities as elliptic functions, J. Stat. Phys. 44 (1986), 713-728.

45. (with R. J. Baxter), Lattice gas generalization of the hard hexagon model. III. q-trinomial coefficients, J. Stat. Phys. 47 (3/4) (1987), 297-330.

46. (with R. J. Baxter and P. J. Forrester), Eight-vertex SOS model and generalized Rogers-Ramanujan-type identities, J. Stat. Phys. 35 (1984), 193-266. 
47. (with A. Berkovich), A trinomial analogue of Bailey's lemma and $N=2$ superconformal invariance, Comm. in Math. Physics 192 (1998), 245-260.

48. (with D. Crippa and K. Simon), q-series arising from the study of random graphs, S.I.A.M. J. Discrete Math. 10 (1) (1997), 41-56.

49. and F. G. Garvan, Dyson's crank of a partition, Bull. Amer. Math. Soc. 18 (1988), 167-171.

50. (with A. and J. Knopfmacher), Engel expansions and the Rogers-Ramanujan identities.

51. _ (with P. Paule and A. Riese), MacMahon's partition analysis III: The omega package.

52. (with P. Paule), MacMahon's partition analysis IV: Summation of series.

53. (with P. Paule), Magic squares and partition analysis, (in preparation).

54. (with O. Warnaar and A. Schilling), An $A_{2}$ Bailey lema and Rogers-Ramanujan-type identities, Accepted for publication J. Amer. Math. Soc..

55. R. Askey, Orthogonal Polynomials and Special Functions, Regional Conf. Series in Applied Math., No. 22, S.I.A.M., Philadelphia, 1975.

56. R. Askey and S. Wainger, A transplantation theorem for ultraspherical coefficients, Pac. J. Math. 16 (1966), 393-405.

57. W. N. Bailey, Generalized Hypergeometric Series, 1935, Cambridge, London and New York (reprinted: Hafner, New York, 1964).

58. _ Identities of the Rogers-Ramanujan type, Proc. London Math. Soc. (2) 50 (1949), 1-10.

59. E. A. Bender and D. E. Knuth, Enumeration of plane partitions, J. Comb. Th., 13 (1972), 40-54.

60. F. Dyson, Three identities in combinatory analysis, J. London Math. Soc. 18 (1943), 35-39.

61. F. J. Dyson, Some guesses in the theory of partitions, Eureka (Cambridge) 8 (1944), 10-15.

62. F. J. Dyson, Mappings and symmetries of partitions, J. Comb. Th. (A).

63. F. Garvan, D. Kim and D. Stanton, Cranks and t-cores, Inv. Math. 101 (1990), 1-17.

64. H. Göllnitz, Einfache Partitionen, (unpublished), Diplomarbeit, W. S. 1960, Göttingen, $65 \mathrm{pp}$.

65. __ Partitionen mit Differenzenbedingungen, Dissertation, Göttingen, 1963, ii + $62 \mathrm{pp}$.

66. _ Partitionen mit Differenzenbedingungen, J. Reine. Angew. Math. 225 (1967), 154-190.

67. B. Gordon, A combinatorial generalization of the Rogers-Ramanujan identities $\mathbf{8 3}$ (1961), 393-399.

68. __ Some continued fractions of the Rogers-Ramanujan type, Duke Math. J. 31 (1965), 741-748.

69. _ A proof of the Bender-Knuth conjecture, Pac. J. Math. 108 (1983), 99-113.

70. H. W. Gould, Combinatorial Identities, West Virginia University, Morgan.

71. W. Hahn, Über Orthogonalpolynome, die q-Differenzengleichungen genügen, Math. Nachr. Berlin 2 (1949), 4-34.

72. G. H. Hardy and E. M. Wright, Introduction to the Theory of Numbers, 4th ed., Oxford University Press, London and New York, 1960.

73. D. Hickerson, A proof of the mock theta conjectures, Inv. Math 94 (1988), 639-660.

74. _ On the seventh order mock theta functions, Inv. Math. 94 (1988), 661-677.

75. W. J. Holman III, L. C. Biedenharn and J. D. Louck, On hypergeometric series well-poised in $S U(n)$, S.I.A.M. J. Math. Anal. 7 (1976), 529-541. 
76. I. G. Macdonald, Affine root systems and Dedekind's $\eta$-function, Inv. Math. 15 (1972), 91-143.

77. 1007.

78. S. Milne, A q-analog of hypergeometric series well-poised in $S U(n)$ and invariant G-functions, Adv. Math..

79. H. Rademacher, Lectures on Analytic Number Theory, Tata Institute, Bombay, 19541955.

80. S. Ramanujan, Collected Papers, Cambridge University Press, London and New York, 1927 (Reprinted: Chelsea, New York 1962).

81. I. Schur, Ein Beitrag zur additiven Zahlentheorie und zur Theorie der Kettenbrüche, S. B. Preuss, Akad. Wiss. Phys.-Math. Kl. 1917, pp. 302-321. (Reprinted in I. Schur, Gesammelte Abhandlungen, Vol. 2, pp. 117-136, Springer, Berlin, 1973).

82. W. Schwarz, Review 1179, Math Reviews 32 (1966), 202-203.

83. A. Selberg, Über einige arithmetische Identitäten, Avhl. Norske Vid. 8 (1936), 23.

84. G. N. Watson, The final problem, J. London Math. Soc. 11 (1936), 55-80.

85. _ The mock theta functions (2), Proc. London Math. Soc. (2) 42 (1937), 274304.

86. D. Zeilberger and D. M. Bressoud, A proof of Andrews' q-Dyson conjecture, Discrete Math. 54 (1985), 201-224. 